\title{
A Syndrome Definition Validation Approach for Zika Virus
}

\author{
Dino P. Rumoro', Shital C. Shah*1, Marilyn M. Hallock', Gillian S. Gibbs", \\ Gordon M. Trenholme ${ }^{1}$ and Michael J. Waddell ${ }^{2}$
}

${ }^{1}$ Emergency Medicine, Rush University Medical Center, Chicago, IL, USA; ²Pangaea Information Technologies, Chicago, IL, USA

\section{Objective}

To develop and validate a Zika virus disease syndrome definition within the GUARDIAN (Geographic Utilization of Artificial Intelligence in Real-Time for Disease Identification and Alert Notification) surveillance system.

\section{Introduction}

In 2016, the World Health Organization declared Zika virus a global public health emergency. Zika infection during pregnancy can cause microcephaly and other fetal brain defects. To facilitate clinicians' ability to detect Zika, various syndrome definitions have been developed.

\section{Methods}

To create and validate a detailed syndrome definition for Zika, we utilized the literature based methodology developed and documented by GUARDIAN researchers. ${ }^{1,2}$ The syndrome definition utilized clinical signs and symptoms that were documented in historical Zika cases.

A testing sample of 1000 randomly selected emergency department cases (i.e., true negative cases) and 200 synthetically generated cases (i.e., true positive cases) was created. These 1,200 sample cases were evaluated by the GUARDIAN surveillance system to determine the probability of matching the Zika syndrome definition. A probability of $\geq 90 \%$ was utilized to designate positive Zika cases.

We identified the main signs and symptoms contributing to the identification of Zika cases and conducted statistical performance metrics. Clinical review of the false positive and false negative cases along with a sample of true positive and true negative cases was conducted by a board certified emergency physician.

\section{Results}

The Zika syndrome definition was developed with eleven articles (six used for developing the syndrome definition, and five used for testing the definition). The sample size for these articles was between 1 and 72 positive Zika cases, with a total of 139 cases across the 11 articles. The article with the most number of Zika cases was based on pregnant women with rash. The publication timeframe for the articles was from 1962 to 2016 . Some of the main signs and symptoms from the historical cases that contribute to the Zika syndrome definition are presented in Table 1 . The initial results for the sample testing data showed accuracy, sensitivity, and specificity were $94.7 \%, 93 \%$, and $95 \%$ respectively. There were a total of 14 false negative and 50 false positive cases.

\section{Conclusions}

The initial Zika syndrome definition utilized by the GUARDIAN surveillance system contains similar signs and symptoms to the current CDC case definition, but also includes additional signs and symptoms such as pruritus/itching, malaise/fatigue/generalized weakness, headache, retro-orbital pain, myalgia/muscle pain, and lymphadenopathy In addition, the GUARDIAN system provides the relative importance of identified signs and symptoms and allows for proactive surveillance of emergency department patients in real-time.
Though we did not include epidemiologic risk factors, such as travel to an infected region or contact with an infected person in the syndrome definition, GUARDIAN has above $90 \%$ sensitivity and specificity. Thus, inclusion of epidemiologic risk factors would further enhance the early detection of Zika, when used with the appropriate high risk population.

Table 1. Main signs and symptoms of Zika syndrome definition

\begin{tabular}{|c|c|}
\hline Signs and Symptoms & Relative Importance (\%) \\
\hline Rash & 90.2 \\
\hline Macular Rash & 40.2 \\
\hline Maculopapular Rash & 37.0 \\
\hline Pruritus/Itching & 76.1 \\
\hline Arthralgia/Joint Pain* & 55.4 \\
\hline Conjunctivitis* $^{*}$ & 50.0 \\
\hline Malaise/Fatigue/Generalized Weakness & 47.8 \\
\hline Headache & 46.7 \\
\hline Retro-orbital Pain & 38.0 \\
\hline Myalgia/Muscle Pain & 38.0 \\
\hline Fever* $^{*}$ & 35.9 \\
\hline Lymphadenopathy & 32.6 \\
\hline
\end{tabular}

*Signs and symptoms included in the Centers for Disease Control and Prevention (CDC)'s Zika clinical case definition.

Keywords

Zika Virus; GUARDIAN; Syndrome Definition

\section{Acknowledgments}

GUARDIAN is funded by the US Department of Defense, Telemedicine and Advanced Technology Research Center, Award numbers W81XWH-09-1-0662 and W81XWH-11-1-0711.

\section{References}

1. Silva J, Rumoro D, Hallock M, Shah S, Gibbs G, Waddell M, Thomas K. Disease profile development methodology for syndromic surveillance of biological threat agents. Emerging Health Threats Journal. 2011; 4(11129).

2. Silva J, Shah S, Rumoro D, Hallock M, Gibbs G, Waddell M. A Novel Syndrome Definition Validation Approach for Rarely Occurring Diseases. Online Journal of Public Health Informatics. 2013; 5(1).

*Shital C. Shah

E-mail:Shital_Shah@rush.edu 